\title{
Genetic Structure of Colletotrichum gloeosporioides sensu lato Isolates Infecting Papaya Inferred by Multilocus ISSR Markers
}

\author{
Sephra N. Rampersad
}

The University of the West Indies, Department of Life Sciences, St. Augustine, Trinidad and Tobago. Accepted for publication 4 October 2012.

\begin{abstract}
Rampersad, S. N. 2013. Genetic structure of Colletotrichum gloeosporioides sensu lato isolates infecting papaya inferred by multilocus ISSR markers. Phytopathology 103:182-189.

Colletotrichum gloeosporioides sensu lato is widely distributed throughout temperate and tropical regions and causes anthracnose disease in numerous plant species. Development of effective disease management strategies is dependent on, among other factors, an understanding of pathogen genetic diversity and population stratification at the intraspecific level. For 132 isolates of $C$. gloeosporioides sensu lato collected from papaya in Trinidad, inter-simple-sequence repeat-polymerase chain reaction (ISSRPCR) generated 121 polymorphic loci from five ISSR primers selected from an initial screen of 22 ISSR primers. The mean percentage of polymorphic loci was $99.18 \%$. Bayesian cluster analysis inferred three genetic subpopulations, where group 1 consisted exclusively of isolates collected in the southern part of Trinidad whereas groups 2 and 3, although genetically distinct, were mixtures of isolates collected from both the northern and southern parts of Trinidad. Principal coordinates analysis and un-

weighted pair-group method with arithmetic mean phylogeny were concordant with Bayesian cluster analysis and supported subdivision into the three subpopulations. Overall, the total mean gene diversity was 0.279 , the mean within-population gene diversity was 0.2161 , and genetic differentiation for the Trinidad population was 0.225 . Regionally, northern isolates had a lower gene diversity compared with southern isolates. Nei's gene diversity was highest for group $1(h=0.231)$, followed by group 2 $(h=0.215)$ and group $3(h=0.202)$. Genotypic diversity was at or near maximum for all three subpopulations after clone correction. Pairwise estimates of differentiation indicated high and significant genetic differentiation among the inferred subpopulations (Weir's $\theta$ of 0.212 to 0.325 ). Pairwise comparisons among subpopulations suggested restricted gene flow between groups 1 and 2 and groups 1 and 3 but not between groups 2 and 3 . The null hypothesis of random mating was rejected for all three inferred subpopulations. These results suggest that pathogen biology and epidemiology as well as certain evolutionary factors may play an important role in population substructuring of $C$. gloeosporioides sensu lato isolates infecting papaya in Trinidad.
\end{abstract}

Colletotrichum is a large, diverse genus of haploid Ascomycete fungi. There are 40 to 45 Colletotrichum spp. that are now accepted; however, Colletotrichum gloeosporioides is one of the most ubiquitous fungal species worldwide and has a diverse host range $(7,8,40)$. The fungus is responsible for anthracnose disease in a number of economically important plants species in temperate, tropical, and subtropical regions (3). C. gloeosporioides is considered to be an aggregate or species complex $(8,20)$ whose members include several putative and as-yet-undefined subspecies or intermediate forms (10). Within the taxa, subgroups show overlap in morphology, pathogenicity, and host specificity and display varying levels of genetic homogeneity $(7,10,18)$. According to the phylogenetic species concept, sublineages that cannot be diagnostically separated by phenotype should not be considered to be distinct species (16).

C. gloeosporioides sensu lato can exist in latent infections as a facultative parasite or endophyte and is capable of causing infections in $>470$ host genera, including tropical fruit species such as papaya (Carica papaya L.) (43). Papaya is cultivated in tropical and subtropical countries and is an economically important fruit tree crop that accounts for a substantial share of tropical fruit production in developing countries (46). Globally, Asia and countries of the Pacific region are the major papaya producers

Corresponding author: S. N. Rampersad

E-mail address: sephra.rampersad@sta.uwi.edu

* The $e$-Xtra logo stands for "electronic extra" and indicates that the online version contains one supplemental figure. Figures 2 and 3 appear in color online.

http://dx.doi.org/10.1094/PHYTO-07-12-0160-R

(C) 2013 The American Phytopathological Society followed by South and Central America and Africa (46). In Trinidad, production areas are concentrated in the south $(87 \%$ of production), with scattered cultivations in the north (38). Disease pressure is expected to be higher in cultivations located in the south, with a concomitant increase in fungicide use compared with cultivations in the north. A study conducted by Rampersad (38) concluded that two species, Colletotrichum gloeosporioides sensu lato and $C$. truncatum, are responsible for anthracnose disease of papaya in Trinidad; however, infection is more commonly caused by $C$. gloeosporioides sensu lato. There are no known commercially available papaya cultivars that are resistant to C. gloeosporioides sensu lato and disease management depends on chemical use. Symptoms of infection are usually seen as circular sunken lesions with a gelatinous effect on mature to ripe fruit; pinkish to salmon-colored spores may or may not be present (38).

A molecular approach to characterizing the species complex has been recommended due to limited use of taxonomic characters and because genetic diversity can be more objectively defined using molecular approaches $(6,10)$. However, earlier approaches may have underestimated genetic diversity due to reliance on data from a single gene region that may have lacked sufficient polymorphisms for exploiting genetic variability at the subspecies level. The use of multilocus comparative sequence analysis has been recommended as part of a polyphasic approach to studying genetic diversity of the species complex; however, there is no agreement on which genes would be the most informative for estimating intraspecific genetic diversity as different genes are subject to different rates of substitution and evolution $(6,10)$. Other molecular techniques such as random amplified polymorphic DNA (RAPD), restriction fragment length polymorphism (RFLP), 
and amplified fragment length polymorphism (AFLP) fingerprinting; analysis of A-T-rich DNA sequences in mitochondrial DNA; internal transcribed spacer RFLP polymerase chain reaction (PCR); arbitrarily primed (AP)-PCR; and sequence comparisons of certain nuclear, structural, and metabolic genes have been used to characterize the $C$. gloeosporioides species complex with variable success $(15,18)$.

Multilocus molecular marker technology has been applied for the identification and authentication of a number of plant and animal species and is highly suited to assessing the genetic structure of plant-pathogenic fungi $(9,19,27,29,30,31,39,54)$. One approach, inter-simple-sequence repeat (ISSR)-PCR, is a discriminatory, genome-wide DNA fingerprinting method that involves amplification of a DNA segment flanked by two identical microsatellite repeats but oriented in opposite directions (5). These simple-sequence repeats (SSRs) or microsatellites are hypervariable and are randomly distributed throughout the genome; the number of these repeating motifs will vary according to individual. The evolutionary rate of change within microsatellite regions is significantly higher than for other regions, and it is the hypervariable nature of ISSR markers that enables detection of a higher level of polymorphism compared with other molecular markers $(51,54)$. The main advantages of ISSR-PCR also include low cost, simple operation, high stability and reproducibility, and rapid turnover (51). ISSR markers distinguished $C$. acutatum isolates infecting almond from Australia from isolates in California and Israel $(26,28)$ and allowed subspecific variation among Colletotrichum isolates in Guyana and Brazil $(19,32)$.

Understanding the genetic diversity and population structure of this species complex is important because it will assist in the understanding of pathogen population dynamics, which is useful when breeding for resistance or monitoring the sensitivity of fungicides across a collection of isolates. Genetic structure and gene flow influence the generation and distribution of new genotypes which affect the evolutionary and adaptive potential of the pathogen under changing selection pressures and contribute to population viability and persistence (22-25). Knowledge of the genetic structure of the pathogen will also reveal the relative impact of certain evolutionary forces (e.g., selection, migration, and recombination) on epidemiology which, ultimately, will assist in developing improved integrated pest management strategies. The objectives of this work were to (i) develop appropriate ISSR genetic markers for intraspecific demarcation of C. gloeosporioides sensu lato isolates from papaya from Trinidad and (ii) use these ISSR markers to estimate the genetic diversity and population structure of these $C$. gloeosporioides sensu lato isolates based on measures of gene diversity, genetic differentiation, linkage disequilibrium, gene flow, and cluster analysis.

\section{MATERIALS AND METHODS}

Isolate collection. A country-wide collection from the main papaya production areas in Trinidad was carried out (Table 1) in 2010 and 2011. In total, 132 C. gloeosporioides sensu lato isolates were collected and their identity was confirmed by molecular analysis (38). There were no a priori assumptions about the number of populations or subpopulations; the entire Trinidad sample set was considered in the operational sense (i.e., a

TABLE 1. Colletotrichum gloeosporioides sensu lato isolates from papaya used to determine population structure

\begin{tabular}{lllc}
\hline Number of isolates & \multicolumn{1}{c}{ Year } & County & Region \\
\hline 57 & 2010,2011 & Victoria & South \\
20 & 2011 & St. Patrick & South \\
12 & 2011 & Mayaro & South \\
16 & 2010 & St. Andrew & North \\
27 & 2010 & St. George & North \\
\hline
\end{tabular}

collection of isolates from a specific country). For some comparisons, isolates were grouped according to region of origin (i.e., north and south). The north is considered to consist of locations at the base of the northern range landmark; similarly, the south is considered to consist of locations at the base of the southern range landmark.

ISSR profiling. Total DNA was extracted from monosporic cultures of $C$. gloeosporioides isolates grown in potato dextrose broth for 9 days in the dark using the E.Z.N.A. SP fungal DNA extraction kit (Omega Bio-tek Inc., GA), in accordance with the manufacturer's instructions.

Four C. gloeosporioides isolates were used in a preliminary screen to determine those ISSR primers that enabled generation of polymorphic, reproducible markers that could be used to generate polymorphic DNA fingerprints for all C. gloeosporioides isolates. This screen tested 22 ISSR primers (Integrated DNA Technologies, IA) (Table 2) which were previously reported to produce polymorphic and reproducible DNA fingerprint patterns from other fungi or plant species. In addition to primer screening, optimization of PCR conditions included titration of $\mathrm{MgCl}_{2}$ and DNA concentration and assessment of optimal primer annealing temperature by gradient annealing temperature analysis. For a single 25- $\mu$ l reaction, PCR components (Invitrogen by Life Technologies, CA) were $1 \times$ PCR buffer, $1.5 \mathrm{mM} \mathrm{MgCl}_{2}, 0.2 \mathrm{mM}$ dNTP, $0.5 \%$ Tween-20, bovine serum albumen (Sigma-Aldrich, St. Louis) at $0.4 \mathrm{mg} / \mathrm{ml}, 1 \mathrm{U}$ of Taq DNA Polymerase, and $10 \mu \mathrm{M}$ each primer. Standard PCR amplification conditions were an initial denaturation of $5 \mathrm{~min}$ at $95^{\circ} \mathrm{C}$; followed by 35 cycles of $30 \mathrm{~s}$ at $95^{\circ} \mathrm{C}, 30 \mathrm{~s}$ at 41 to $60^{\circ} \mathrm{C}$, and $90 \mathrm{~s}$ at $72^{\circ} \mathrm{C}$; with a final extension of $5 \mathrm{~min}$ at $72^{\circ} \mathrm{C}$. The PCR reactions were run on a Techne thermocycler (BioRad Laboratories Ltd., Ontario, Canada). PCR products were separated on $1.4 \%$ agarose gels stained with ethidium bromide. A 1-kb and a 100-bp DNA ladder (Invitrogen by Life Technologies) were used as molecular weight markers. PCR reactions were conducted twice to confirm reproducibility of the fingerprints. Those primers that generated reproducible, clearly discernible, polymorphic bands in repeated experiments were selected to subsequently amplify genomic DNA from all isolates. Bands were scored computationally using the GelAnalyzer 2010a software (http://www.gelanalyzer.com). Only bands (size: 100 to 2,500 bases) that could be scored consistently for all samples

TABLE 2. Characteristics of inter-simple-sequence repeat (ISSR) primers screened

\begin{tabular}{|c|c|c|}
\hline ISSR primer ${ }^{\mathrm{a}}$ & $\mathrm{Ta}\left({ }^{\circ} \mathrm{C}\right)^{\mathrm{b}}$ & Primer description \\
\hline$* P 1:(A G)_{8}$ & 43.4 & Anchorless di-nucleotide \\
\hline$* \mathrm{P} 2:(\mathrm{AC})_{8}$ & 47.4 & Anchorless di-nucleotide \\
\hline $\mathrm{P} 3:(\mathrm{CAG})_{5}$ & 47.4 & Anchorless tri-nucleotide \\
\hline P4: $(\mathrm{CAA})_{5}$ & 33.7 & Anchorless tri-nucleotide \\
\hline P5: $(\mathrm{GTG})_{5}$ & 47.4 & Anchorless tri-nucleotide \\
\hline P6: $(\mathrm{CAC})_{5}$ & 47.4 & Anchorless tri-nucleotide \\
\hline P7: $(\mathrm{ACA})_{5}$ & 33.7 & Anchorless tri-nucleotide \\
\hline P8: $(\mathrm{AAC})_{5}$ & 33.7 & Anchorless tri-nucleotide \\
\hline P9: $(\mathrm{CCA})_{5}$ & 54.9 & Anchorless tri-nucleotide \\
\hline P10: $(\mathrm{AAG})_{6}$ & 41.2 & Anchorless tri-nucleotide \\
\hline P11: $(\mathrm{GTC})_{6}$ & 54.9 & Anchorless tri-nucleotide \\
\hline *P12: $(\mathrm{GACA})_{4}$ & 43.4 & Anchorless tetra-nucleotide \\
\hline P13: $(\text { GGAT })_{4}$ & 44.7 & Anchorless tetra-nucleotide \\
\hline P14: $(\mathrm{GACAC})_{3}$ & 44.7 & Anchorless penta-nucleotide \\
\hline$* P 15:(\mathrm{AG})_{8} \mathrm{TG}$ & 48.0 & Di-nucleotide, $3^{\prime}$ anchor present \\
\hline P16: $(\mathrm{AG}){ }_{8} \mathrm{CG}$ & 48.0 & Di-nucleotide, $3^{\prime}$ anchor present \\
\hline P17: $(\mathrm{CT})_{8} \mathrm{G}$ & 48.0 & Di-nucleotide, $3^{\prime}$ anchor present \\
\hline P18: $(\mathrm{CA})_{8} \mathrm{GT}$ & 48.0 & Di-nucleotide, $3^{\prime}$ anchor present \\
\hline P19: $\mathrm{CTC}(\mathrm{GT})_{8}$ & 48.0 & Di-nucleotide, $5^{\prime}$ anchor present \\
\hline *P20: CT(GA) 8 & 48.0 & Di-nucleotide, $5^{\prime}$ anchor present \\
\hline $\mathrm{P} 21: \mathrm{BDB}(\mathrm{ACA})_{5}$ & 48.0 & Tri-nucleotide, $5^{\prime}$ anchor present \\
\hline P22: $\mathrm{GAG}(\mathrm{CAA})_{5}$ & 48.0 & Tri-nucleotide, $5^{\prime}$ anchor present \\
\hline
\end{tabular}


were used, with the assumption that each band represented a distinct locus and amplicons sharing the same molecular weight were considered to be the same locus.

Data analysis. Amplified DNA fragments were transformed into a binary character matrix $(1=$ presence, $0=$ absence $)$. Bayesian clustering implemented by STRUCTURE 2.3 was first used to determine the probability that each isolate belonged to a distinct subpopulation or genetic cluster assuming admixture and correlated allele frequency $(13,14,37)$. The model was run for 50,000 Markov chain Monte Carlo iterations after an initial burnin period of 10,000 generations from $K=1$ to $K=10$ for all 132 isolates. The models implemented in this version of STRUCTURE can resolve genetic structure at lower levels of divergence with less data than other models, and they are not prone to inferring structure when it is absent (false signals) (12). The ad hoc $K$ statistic $(\Delta K)(12)$ was used to predict the most likely number of populations $(K)$ in the data after 20 runs. The modal value of $\Delta K$ is a quantity based on the second-order rate of change with respect to $K$. After determining the number of inferred subpopulations or clusters, GenAlEx version 6.3, Genetic Analysis in Excel (36) was used to produce a genetic distance matrix; calculation of pairwise genetic distances for binary data followed the method of Huff et al. (17). Principal coordinates analysis (PCoA) was also carried out for separation of the isolates.

Nei's gene diversity $(h)$ (33) allele frequencies and pairwise comparisons of genetic diversity for each inferred subpopulation were calculated using POPGENE version 1.32 (53) with 1,000 permutations. Genetic diversity was estimated according to McDonald (24); for haploid fungi that are assessed with dominant markers, the theoretical threshold of genetic diversity would be 0.50 , assuming that both alleles are present with the same frequency. Weir and Cockerham's $\theta(49,50)$, which is comparable with Wright's $F_{\mathrm{ST}}$, was calculated using MULTILOCUS version 1.32b (Department of Biology, Imperial College at Silwood Park, $\mathrm{UK}$ ). The $\theta$ statistic ranges from 0 (for no population differentiation) to 1 (complete differentiation where populations are fixed for different alleles). Pairwise gene flow $\left(N_{\mathrm{m}}\right)$ was estimated for all subpopulations, where $N_{\mathrm{m}}<1$ is indicative of low levels of gene flow; $N_{\mathrm{m}}=1$ means that effects of drift are exactly counterbalanced by the effects of gene flow, so that populations neither diverge nor converge; and $N_{\mathrm{m}}>1$ suggests high levels of gene flow (22).
Linkage disequilibrium as an indication of random mating was calculated and tested for significance with 1,000 randomizations using MULTILOCUS; measures of gametic disequilibrium tested were (i) the proportion of compatible pairs of loci, (ii) the index of association $\left(I_{\mathrm{A}}\right)$, and (iii) a standardized alternative of the $I_{\mathrm{A}}$ (2). The null hypothesis for this test is that there is random association among alleles at different loci and $I_{\mathrm{A}}=0$; the null hypothesis for random mating is rejected where $I_{\mathrm{A}}>0$.

For phylogenetic assignments, candidate isolates from each of the three inferred clusters were selected and the final data set for analysis consisted of 44 isolates (isolates 1 to 28 were from the south and isolates 29 to 44 were from the north). An unweighted pair group method with arithmetic mean (UPGMA) (42) phylogram was generated using Molecular Evolutionary Genetic Analysis software, version 5 for Windows (44). Bootstrapping was performed with WinBoot software using Dice's coefficient with 10,000 bootstrap (BS) resamplings (52).

\section{RESULTS}

ISSR markers. Only those individuals that produced a polymorphic banding pattern for the five primers (Supplemental Figure S1) and for which the interpretation of the banding pattern was unequivocal were retained in the final data set. Fragments with the same molecular weight were considered to be the same locus. In total, 121 polymorphic loci were generated from ISSRPCR using the five ISSR primers selected from a screen of 22 ISSR primers (Table 2). The highest number of polymorphic bands (PBs) was produced for P2 (an anchorless di-nucleotide, 35 PBs), followed by P15 (a 3'-anchored di-nucleotide, 23 PBs), P1 (an anchorless di-nucleotide, 22 PBs), P12 (a tetra-nucleotide, 22 PBs), and P20 (a 5'-anchored di-nucleotide, 20 PBs). A high level of genetic variation was obtained using ISSR markers; the mean percentage of polymorphic loci was $99.18 \%$. Isolates from the north $(57.4 \%)$ had a lower level of polymorphism than those from the south $(92.6 \%)$ (Table 3$)$.

Population stratification. Assignment of isolates was carried out by Bayesian clustering implemented by STRUCTURE software. Posterior probabilities calculated by STRUCTURE show population structure at $K=3$ genetic groups or clusters (Fig. 1). The overall estimated proportion of membership of the samples to each of the three inferred genetic groups was 19.2, 40.4, and

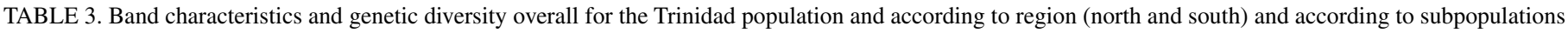

\begin{tabular}{|c|c|c|c|c|c|c|}
\hline Index & Overall & Northern region & Southern region & Group 1 & Group 2 & Group 3 \\
\hline \multicolumn{7}{|l|}{ Number of } \\
\hline Isolates & 132 & 48 & 84 & 30 & 51 & 51 \\
\hline Different bands & 121 & 71 & 113 & 83 & 88 & 79 \\
\hline Bands (frequency $\geq 5 \%$ ) ${ }^{\mathrm{a}}$ & 97 & 71 & 98 & 83 & 88 & 79 \\
\hline Private bands ${ }^{b}$ & 121 & 71 & 113 & 19 & 15 & 2 \\
\hline Local bands $(\leq 25 \%)^{\mathrm{c}}$ & 0 & 0 & 0 & 0 & 0 & 0 \\
\hline Local bands $(\leq 50 \%)^{\mathrm{d}}$ & 0 & 0 & 0 & 0 & 0 & 0 \\
\hline \multicolumn{7}{|l|}{ Alleles } \\
\hline Number different (Na) & 1.984 & 1.156 & 1.852 & 1.352 & 1.443 & 1.270 \\
\hline $\mathrm{SE}$ of mean $\mathrm{Na}^{\mathrm{e}}$ & 0.016 & 0.089 & 0.048 & 0.085 & 0.082 & 0.086 \\
\hline Number effective $(\mathrm{Ne})^{\mathrm{f}}$ & 1.431 & 1.332 & 1.435 & 1.385 & 1.348 & 1.332 \\
\hline $\mathrm{SE}$ of mean $\mathrm{Ne}$ & 0.028 & 0.302 & 0.029 & 0.032 & 0.030 & 0.031 \\
\hline Mean $h^{\mathrm{g}}$ & 0.270 & 0.200 & 0.268 & 0.231 & 0.016 & 0.202 \\
\hline SE of mean $h$ & 0.013 & 0.017 & 0.015 & 0.017 & 0.202 & 0.017 \\
\hline Mean $\mathrm{u} h^{\mathrm{h}}$ & 0.276 & 0.213 & 0.278 & 0.257 & 0.017 & 0.214 \\
\hline $\mathrm{SE}$ of mean $\mathrm{u} h$ & 0.014 & 0.019 & 0.015 & 0.019 & 0.214 & 0.018 \\
\hline Polymorphism (\%) & 99.18 & 57.38 & 92.62 & 67.21 & 72.13 & 62.30 \\
\hline
\end{tabular}

a Number of different bands with a frequency $>5 \%$.

b Number of bands unique to a single population.

c Number of locally common bands (frequency $>5 \%$ ) found in $\leq 25 \%$ populations.

d Number of locally common bands (frequency $>5 \%$ ) found in $\leq 50 \%$ populations.

e Standard error (SE) of mean number of different alleles.

${ }^{\mathrm{f}}$ Number of effective alleles $=1 /\left(\mathrm{p}^{\wedge} 2+\mathrm{q}^{\wedge} 2\right)$ (where, for haploid binary data, $\mathrm{p}=$ band frequency and $\left.\mathrm{q}=1-\mathrm{p}\right)$.

$\mathrm{g}$ Gene diversity $=1-\left(\mathrm{p}^{\wedge} 2+\mathrm{q}^{\wedge} 2\right)$.

${ }^{\mathrm{h}}$ Unbiased gene diversity, where $[N /(N-1)] \times h$. 
$40.4 \%$ (Table 3 ) and the assignment of isolates to putative clusters is shown in Figure 2. The inferred genetic structure where $K=3$ is shown in Figure 2 according to an ad hoc $\mathrm{K}$ statistic. Group 1 consisted exclusively of southern isolates whereas groups 2 and 3 were mixtures of southern and northern isolates (Fig. 3). Band statistics and polymorphisms are presented in Table 3 for the three inferred subpopulations.

PCoA was carried out to estimate genetic similarity between individuals to further represent inter-individual and inter-group relationships. The two-dimensional PCoA plot (Fig. 2) indicated that the first and second principal coordinates accounted for 32.68 and $20.41 \%$, respectively, of the total variation. Individuals were positioned into discrete groups; two of these groups consisted of both northern and southern isolates but the third group consisted exclusively of southern isolates. Isolate placement was independent of region (i.e., north and south). Therefore, PCoA results were concordant with Bayesian cluster analysis implemented by STRUCTURE.

Data from the genetic distance matrices were analyzed using an UPGMA algorithm; only the optimal UPGMA tree is presented

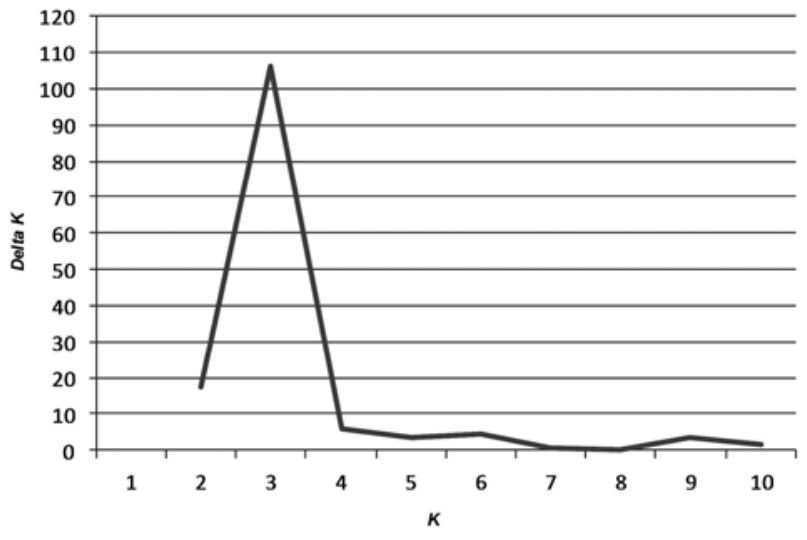

Fig. 1. $\Delta \mathrm{K}$ plot to determine most probable $\mathrm{K}$.
(Fig. 4). Population stratification was reflected in the tree topology, which consisted of three main subdivisions among the isolates. Robust BS support was indicated for separation of group 1 isolates ( $\mathrm{BS}>70 \%)$; however, strong $\mathrm{BS}$ support $(\mathrm{BS}>70 \%)$ for division of isolates into groups 2 and 3 was absent. Overall, the main groups produced were group 1, which consisted exclusively of southern isolates; group 2 and group 3 consisted of a mixture of both northern and southern isolates but these appeared to be distinct subdivisions. Strict clustering of isolates based on region (i.e., north and south) was not observed.

Genotypic and gene diversity. The overall mean gene diversity $\left(H_{\mathrm{T}}\right)$ was 0.279 and the mean within-population gene diversity $\left(H_{\mathrm{S}}\right)$ was 0.2161 . The overall genetic differentiation for the Trinidad population $\left(G_{\mathrm{ST}}\right)$ was 0.225 , which suggests that $22.5 \%$ of the total variation among all isolates in the dataset was within subpopulations and $77.5 \%$ was among subpopulations. Regionally, northern isolates had a lower gene diversity $(h=0.200)$ compared with southern isolates $(h=0.268)$. Group 1 had the highest gene diversity $(h=0.231)$, followed by group $3(h=0.202)$, and group $2(h=0.016)$ had the lowest gene diversity. Pairwise comparisons of Nei's unbiased measures of genetic identity and genetic distance estimated for three inferred subpopulations are given in Table 4. Pairwise estimates of differentiation $(\theta$; below the diagonal in Table 5) indicate high and significant genetic differentiation among the inferred subpopulations. In total, 36 unique multilocus genotypes were identified among all three inferred subpopulations. Within each group, genotypic diversity was 1.000 for group 1, 0.991 for group 2, and 0.961 for group 3 .

Gene flow. Estimates of gene flow $\left(N_{\mathrm{m}}\right)$ among three subpopulations of $C$. gloeosporioides sensu lato isolates based on 121 polymorphic loci are presented in Table 5 (above the diagonal). Pairwise comparisons among subpopulations suggested that there was neither divergence nor convergence between groups 1 and 2 and between groups 1 and 3 , where $N_{\mathrm{m}} \approx 1$. Between groups 2 and $3, N_{\mathrm{m}}$ was 1.858 , even though these two subpopulations were significantly and highly differentiated from each other.

Linkage disequilibrium. For the $I_{\mathrm{A}}$ test, the null hypothesis of random mating $\left(I_{\mathrm{A}}=0\right)$ was rejected for all three inferred sub-

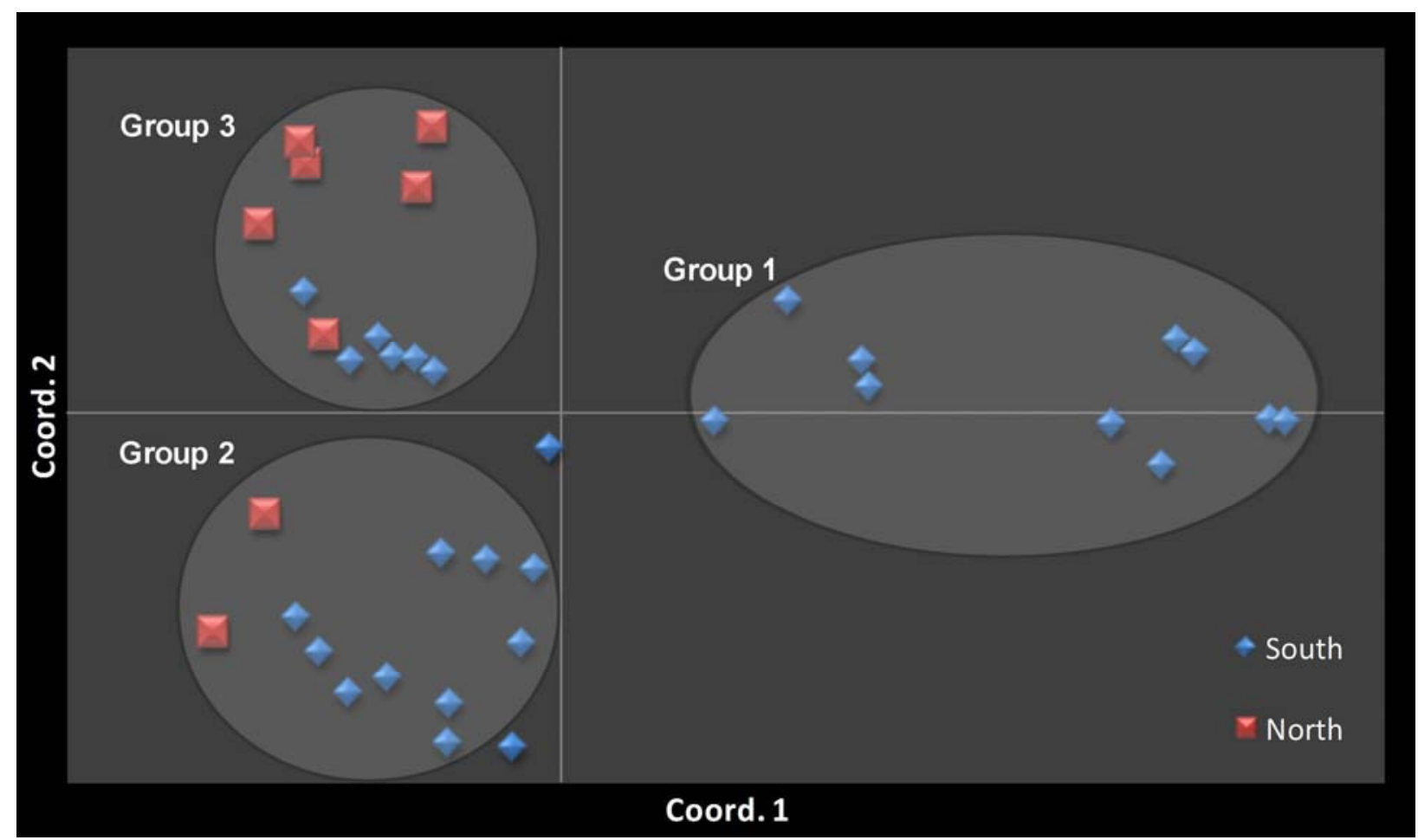

Fig. 2. Two-dimensional plot of the principal coordinates analysis showing patterns of subspecific clustering of Colletotrichum gloeosporioides sensu lato isolates from papaya from Trinidad; three genetic groups were identified. 
populations. The $I_{\mathrm{A}}$ was significantly different from zero $\left(I_{\mathrm{A}}>0\right)$ after 1,000 randomizations for each subpopulation (Table 6). In determining the proportion of phylogenetically compatibly loci pairs, the null hypothesis of random mating was also rejected because significantly more compatible pairs of loci were observed than expected under random mating for each subpopulation. Clonal genotypes were distributed within each subpopulation; to account for over-representation of clonal types, clone-censored data were used to retest the null hypothesis of random mating. The number of individuals in each subpopulation was considerably lower after clone correction; the $I_{\mathrm{A}}$ values were also reduced but were still significantly different from zero $\left(I_{\mathrm{A}}>0\right)$ after 1,000 randomizations for each subpopulation (Table 6).

\section{DISCUSSION}

This is the first study to characterize partitioning of genetic diversity and population structure of $C$. gloeosporioides sensu lato isolates infecting papaya based on a genome-wide multilocus ISSR fingerprinting approach. Population genetic analysis supported subdivision of the Trinidad population into three inferred subpopulations characterized by high levels of gene and genotypic diversity, significant and high levels of genetic differentiation among subpopulations, the possibility of gene flow between two subpopulations (undetermined whether past or present events), no strict regional clustering of isolates, and rejection of the null hypothesis of random mating within the three subpopulations.

Intraspecific variation based on ISSR fingerprinting has been shown to be high in some fungal species and low in others (39). The mechanisms responsible for presence or absence of ISSR markers include absence of a primer-binding site due to mutation in the priming site, strand slippage during DNA polymerization due to template instability, insertions or deletions that might increase or decrease amplified fragment length sufficiently for it to be scored as a separate locus, and structural rearrangements of the chromosome that prevent polymerization. Further, the resolving power of ISSR fingerprinting is also dependent on the distribution and availability of complementary primer-binding sites in the target genome, in addition to the size of the fragments flanked by these ISSR primers which must be within amplifiable distance of each other. The base composition of the ISSR primers affects fingerprint quality because primers with high GC content $(>50 \%)$ result in background noise and nonhomologous co-migrating fragments whereas pure A or T primers will result in smears of amplification products (54). Optimizing primer annealing temperature and other reaction conditions may serve to mitigate these effects.

Microsatellite loci evolve at different rates and the variation in repeat number increases as a result of multi-step mutations (11). Regardless of the type of mutational process that led to differential primer binding in ISSR fingerprinting, there may be some bias in the mutation rate. Repeat length and base composition affect mutation rate (i.e., di-nucleotide repeats mutate faster than tri-nucleotide repeats, and sequences with a high AT content mutate faster than those with a high GC content) (40). There may also be a restriction on the number of repeats which may be related to maintaining genome size and chromosomal stability (9).

Many Ascomycete fungi engage mixed reproduction strategies of clonal propagation (mitosporic spore production) and recombination (meiosporic spore production) (45). The reproductive mode affects pathogen fitness in terms of spread and persistence and ultimately affects the genetic structure, which may vary in time and space for a given species $(28,45)$. C. gloeosporioides sensu lato has a sexual or perfect stage (teleomorph: Glomerella cingulata) but it is not always present in culture or under natural conditions. This study rejected the null hypothesis of random mating; however, perithecia were observed in most colonies $(82.3 \%)$ grown in vitro and which were used in the analysis. Milgroom (28) recommended a direct assessment of the potential for recombination based on the presence of sexual structures in
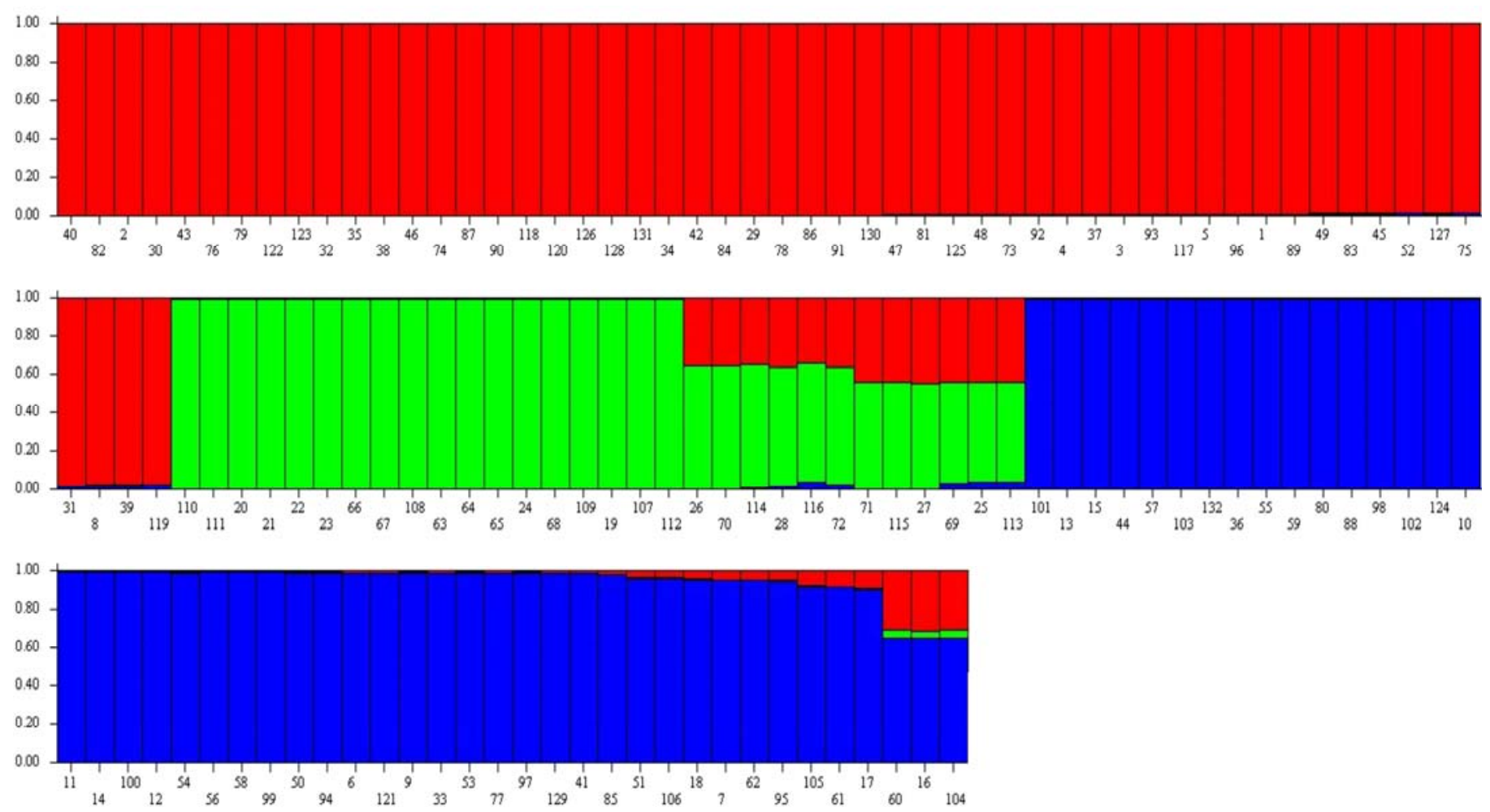

Fig. 3. Relative proportion of the genome of an individual originating from each inferred population (quantitative clustering method) and assignation to clusters. Different shadings represent different genetic groups; each column represents an individual isolate, and the height of the column segments shows the probability of assignment of this isolate to a particular genetic group. The height of each shaded region within an individual bar is the measure of proportional affiliation. Individuals with membership coefficients of $\mathrm{q}_{i} \geq 0.7$ were assigned to a specific genetic cluster. 
culture and under natural conditions. There was also evidence of high genotypic diversity (at or near maximum for the three inferred subpopulations), typical of recombining populations (28). A departure from random mating can only be proposed if all other causes of deviations from random mating (i.e., selection, genetic drift, mutation, epigenetic effects, and physical linkage between loci) have been discounted $(22-25,28,29)$. It is also difficult to determine how often recombination occurs, or whether the identified genetic structure was influenced by past, present, or parasexual recombination events (45).

Strict regional separation of isolates into distinctly isolated subpopulations was not observed because there was evidence of admixture between northern and southern isolates. There was also evidence of gene flow between groups 2 and 3 but whether this was due to recent or past migration events cannot be determined. Mechanisms that enable gene flow may act randomly and may be a result of a combination of (i) anthropogenic activities, including movement and exchange of infected seed and planting material; (ii) the process of extinction and recolonization (which is common to most agroecosystems because of seasonal cropping regimes), which means that most fungal pathosystems are not in evolutionary equilibrium (i.e., a balance between genetic drift and migration); and (iii) alternate hosts outside of the growing season that may allow certain genotypes to persist and undergo expansion in the field $(22-25,28,29)$. Results indicated that group 1, which consisted exclusively of southern isolates, exhibited higher

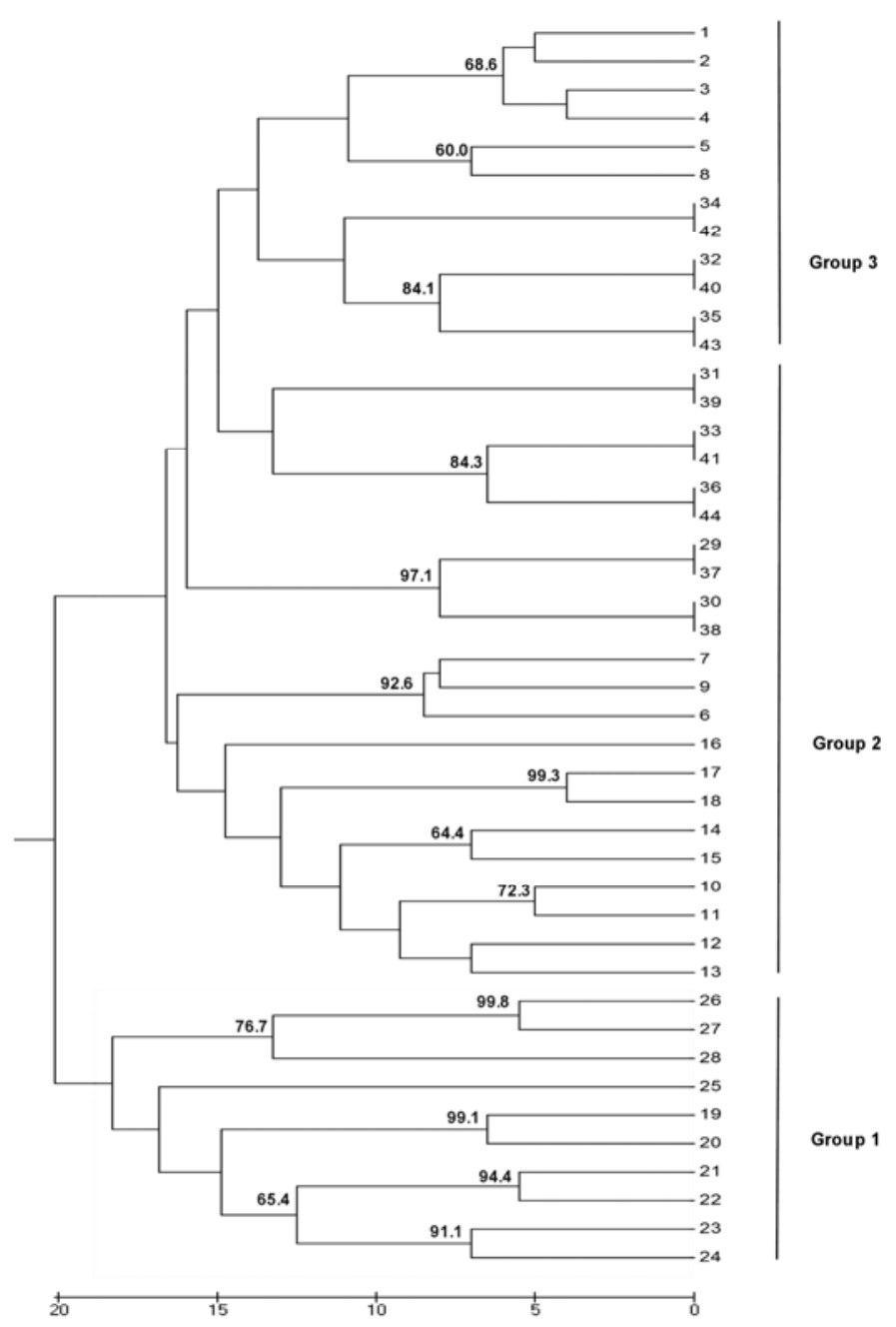

Fig. 4. Unweighted pair group method with arithmetic mean phylogram showing the optimal tree. The tree is drawn to scale, with branch lengths in the same units as those of the evolutionary distances used to infer the phylogenetic tree. Only bootstrap scores $\geq 60.0 \%$ are shown. significant differentiation and restricted gene flow than the other two subpopulations. Group 1 may contain individual members that were recently introduced to Trinidad or may constitute a unique or specially adapted ecological group. These southern isolates may possess novel genotypes with biologically important character combinations. Tracking marked isolates, in an effort to (i) determine the potential risk these genotypes might pose to management strategies and (ii) evaluate differences in cultural or management strategies in the south that may have selected for such isolates in the first place, might prove useful to further understanding the epidemiology of this disease in papaya in Trinidad.

With respect to ISSR polymorphisms between northern and southern isolates, the relative differences observed strictly for ISSR profiles may be due to differences in the number of isolates used for each region (89 southern isolates and 43 northern isolates). These differences, however, were not statistically adequate to separate the isolates into northern and southern subpopulations when more advanced analyses were applied. This underlines the importance of treating the data set without any assumption of substructuring or admixture prior to analysis. STRUCTURE analysis, PCoA, and, to a lesser degree of robustness, the UPGMA algorithm all indicated admixture between northern and southern isolates because there was no distinct northern population.

In this study, there were high levels of genetic differentiation among the inferred subpopulations. In fungal pathogens with mixed reproductive strategies, as for $C$. gloeosporioides sensu lato, recombination and selection can work together to sustain a particular genotype that offers a positive selective advantage, which can allow for a high level of genetic differentiation among subpopulations $(22,28,29)$. An intermediate model of population structure, referred to as an "epidemic" model by Smith et al. (41), is characterized by initial recombination events followed by clonal expansion of one or a few highly successful individuals through multiple successive asexual cycles that may lead to linkage disequilibrium. Although the model was initially suggested for bacteria, it is applicable to many plant fungal pathosystems (28). It is likely that the genetic structure of $C$. gloeosporioides sensu lato isolates infecting papaya is consistent with this model, based on the results of this study. Additionally, genetic differentiation may have progressed to subdivision of the Trinidad population into three inferred subpopulations; however, no locus was

TABLE 4. Measures of pairwise comparisons of Nei's unbiased measures of genetic identity and genetic distance estimated for three inferred subpopulations of Colletotrichum gloeosporioides sensu lato isolates from papaya from Trinidad ${ }^{\mathrm{a}}$

\begin{tabular}{lccc}
\hline Subpopulation & Group 1 & Group 2 & Group 3 \\
\hline Group 1 & $\ldots$ & 0.862 & 0.863 \\
Group 2 & 0.148 & $\ldots$ & 0.925 \\
Group 3 & 0.148 & 0.078 & $\ldots$ \\
\hline
\end{tabular}

a Nei's genetic identity (above diagonal) and genetic distance (below diagonal).

TABLE 5. Pairwise estimates of differentiation $(\theta)$ and gene flow $\left(N_{\mathrm{m}}\right)$ among three subpopulations of Colletotrichum gloeosporioides sensu lato isolates based on 121 polymorphic loci ${ }^{\mathrm{a}}$

\begin{tabular}{lccc}
\hline Subpopulation & Group 1 & Group 2 & Group 3 \\
\hline Group 1 & $\ldots$ & 1.097 & 1.043 \\
Group 2 & $0.314^{*}$ & $\ldots$ & 1.858 \\
Group 3 & $0.325^{* *}$ & $0.212^{* *}$ & $\ldots$ \\
\hline
\end{tabular}

a Weir's $\theta$ (49) is comparable with Wright's $F_{\mathrm{ST}}$ statistic and was estimated according to $\widehat{D}_{l}=\sum_{u=1}^{k} P_{l u}^{2}$ for all 121 loci and averaged, where $P_{l u}$ is the frequency of the alleles $u$ at the $l$ th locus, and $n$ is the number of individuals sampled. $P$ values were estimated with 1,000 randomizations; values calculated were significantly different from zero at $P<0.001(*)$ and $P<0.0001$ $(* *)$, which indicated high and significant subpopulation differentiation. Weir's $\theta$ (below diagonal) and $N_{\mathrm{m}}$ (above diagonal). 
TABLE 6. Estimates of linkage disequilibrium for three inferred subpopulations of Colletotrichum gloeosporioides sensu lato isolates from papaya from Trinidad

\begin{tabular}{|c|c|c|c|c|c|c|}
\hline Subpopulation & $N^{\mathrm{a}}$ & Genotypic diversity $^{b}$ & $\operatorname{PrCL}^{\mathrm{c}}$ & $I_{\mathrm{A}}{ }^{\mathrm{d}}$ & $\bar{r}_{d}^{\mathrm{e}}$ & $P^{\mathrm{f}}$ \\
\hline Group 1 & 30 & 0.931 & 0.900 & 6.052 & 0.075 & $<0.01$ \\
\hline Group 2 & 51 & 0.946 & 0.856 & 4.577 & 0.054 & $<0.01$ \\
\hline Group 3 & 51 & 0.925 & 0.915 & 4.628 & 0.063 & $<0.01$ \\
\hline Group $1 \mathrm{cc}^{\mathrm{g}}$ & 10 & 1.000 & 0.900 & 2.642 & 0.033 & $<0.001$ \\
\hline Group 2cc & 15 & 0.991 & 0.874 & 2.615 & 0.034 & $<0.001$ \\
\hline Group 3cc & 18 & 0.961 & 0.916 & 3.024 & 0.041 & $<0.001$ \\
\hline
\end{tabular}

a Number of samples.

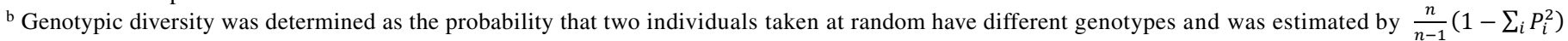
where $P_{i}$ is the frequency of the $i$ th haplotype and $n$ is the number of individuals sampled. This value is 0 if every individual is the same and 1 if every individual is different (MULTILOCUS, version 1.32b).

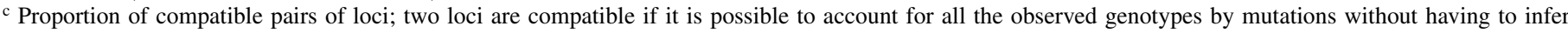
homoplasy (reversals, parallelisms, or convergences) or recombination, such that the two loci define a phylogeny with a consistency index of 1 (MULTILOCUS, version $1.32 \mathrm{~b}$ ).

${ }^{\mathrm{d}}$ Index of association $\left(I_{\mathrm{A}}\right)$ of alleles at all loci (2).

e Alternate standardized index of association $\left(\bar{r}_{d}\right)$ that is independent of loci number (2).

f $P$ values were estimated from 1,000 randomizations and are identical for monontically related statistics $I_{\mathrm{A}}$ and $\left(\bar{r}_{d}\right)$.

g Clone-corrected data (cc); one isolate represented each unique multilocus genotype.

fixed for alternative alleles in any of the three subpopulations, which indicates that the isolates were of the same phylogenetic species (45).

There was evidence of high gene diversity among subpopulations of C. gloeosporioides sensu lato isolates infecting papaya in Trinidad. Gene diversity is not influenced by reproductive mode because fungi that reproduce clonally may have as many alleles as those that undergo recombination (23). Factors other than recombination can affect genetic diversity (4). High genetic diversity may be a function of population size where, under neutrality, larger populations maintain higher levels of gene diversity; older populations have greater gene diversity compared with a recently colonized habitat, especially if this population was founded by only a few colonizers. In an older population, sufficient time has passed to allow mutational events to introduce new genetic variants and for genetic drift to increase the frequencies of these alleles to quantitative levels. McDonald (23) indicated that isolates located at or near the center of origin from which that species originates would have a higher level of gene diversity than isolates at other locations because the original population is older. The center of origin for papaya is considered to be the Caribbean coast of Central America and there are records of papaya seed distribution throughout the Caribbean since the 16th century $(35,46)$. Unique $C$. gloeosporioides genotypes may occupy particular geographical areas which may be associated with host coevolution and adaptation (8). Weeds et al. (48) demonstrated that genetic diversity of $C$. gloeosporioides sensu lato isolates is large where native or naturalized host species occur compared with locations where the host species has been recently introduced.

Dominant marker systems (e.g., AFLP and RAPD) have only two alleles per locus and, therefore, have a maximum theoretical value for gene diversity of 0.50 , which occurs when both alleles are present at equal frequency (25). In addition, in multilocus studies, the investigated loci have usually been preselected for high levels of polymorphism (34); thus, it is not appropriate to compare gene diversity for the same species if different marker systems were used (24). However, simply as a matter of course, a comparison of relevant studies revealed that highly differentiated clonal lineages in the absence of significant recombination have been reported for C. gloeosporioides sensu lato infecting Stylosanthes spp. in Australia (21). C. gloeosporioides sensu lato infecting yam in Nigeria (1), was described as a single panmictic population with low intrapopulation differentiation $\left(G_{\mathrm{ST}}=0.05\right)$ and high genetic diversity $\left(H_{\mathrm{T}}=0.27\right)$; high genotypic diversity (near maximum) and evidence of unrestricted gene flow $\left(N_{\mathrm{m}}=\right.$ 8.85 ), as inferred by 51 microsatellite loci, were reported. Conversely, there was significant and high genetic differentiation and gene diversity of subpopulations of $C$. gloeosporioides sensu lato infecting strawberry in the United States based on 40 RAPD and microsatellite markers (47). In both studies, recovery of the sexual stage of the fungus was indicative of recombination.

It is worth noting that these explanations cannot be concluded with finality without further investigations. The critical number of individuals in a population, number of markers and type of marker system, number of effective loci, as well as other parameters needed for these analyses, have not been universally resolved and data are subject to the limitations of many of the statistical tests that are used to determine population structure. These parameters may, in fact, be technique specific, notwithstanding the dominant or co-dominant nature of the genetic markers (34).

It is important to understand the diversity of plant pathogen populations in various ecosystems. Areas of high biodiversity may serve as a source for the emergence of new genotypes with novel biological characteristics, including changes in pathogen fitness or resistance to certain fungicides. Long-term, stable disease management in addition to breeding for resistance and development of resistant transgenics depend on a quantitative assessment of population substructuring of the pathogen. Resistance screening and fungicide baseline monitoring should be against a wide range of genotypes in the pathogen population in order to represent the full spectrum of individuals in a given population. Management strategies would have to focus on all three subpopulations in Trinidad rather than against individual isolates or selected genotypes. Durability of host plant resistance is negatively correlated to the evolutionary potential of target pathogens; hence, understanding the factors that promote the evolutionary success of pathogen populations and the rate of local adaptation is important.

\section{ACKNOWLEDGMENTS}

This work was supported by The University of the West Indies, St. Augustine, Campus Research and Publications Grant (grant number CRP.3.NOV11.8). I thank R. Rampersad for field assistance.

\section{LITERATURE CITED}

1. Abang, M. M., Asiedu, R., Hoffmann, P., Wolf. G. A., Mignouna, H. D., and Winter, S. 2006. Pathogenic and genetic variability among Colletotrichum gloeosporioides isolates from different yam hosts in the agroecological zones in Nigeria. J. Phytopathol. 154:51-61.

2. Agapow, P.-M., and Burt, A. 2001. Indices of multilocus linkage disequilibrium. Mol. Ecol. Notes 1:101-102.

3. Bailey, J. A., and Jeger, M. J. 1992. Colletotrichum: Biology, Pathology and Control. CAB International, Wallingford, UK.

4. Bennett, R. S., Milgroom, M. G., and Bergstrom, G. C. 2005. Population structure of seedborne Phaeosphaeria nodorum on New York wheat. Phytopathology 95:300-305.

5. Brown, J. K. M. 1996. The choice of molecular marker methods for 
population genetic studies of plant pathogens. New Phytol. 133:183-195.

6. Cai, L., Hyde, K. D., Taylor, P. W. J., Weir, B. S., Waller, J., Abang, M. M., Zhang, J. Z., Yang, Y. L., Phoulivong, S., Liu, Z. Y., Prihastuti, H., Shivas, R. G., McKenzie, E. H. C., and Johnston, P. R. 2009. A polyphasic approach for studying Colletotrichum. Fungal Divers. 39:183-204.

7. Cannon, P. F., Bridge, P. D., and Monte, E. 2000. Linking the past, present and future of Colletotrichum systematics. Pages 1-20 in: Colletotrichum: Host Specificity, Pathology, and Host-Pathogen Interaction. D. Prusky, S. Freeman, and M. B. Dickman, eds. American Phytopathological Society, St. Paul, MN.

8. Cannon, P. F., Buddie, A. G., and Bridge, P. D. 2008. The typification of Colletotrichum gloeosporioides. Mycotaxon 104:189-204.

9. Chen, F., Goodwin, P. H., Khan, A., and Hsiang, T. 2002. Population structure and mating-type genes of Colletotrichum graminicola from Agrostis palustris. Can. J. Microbiol. 48:427-436.

10. Damm, U., Baroncelli, R., Cai, L., Kubo, Y., O’Connell, R., Weir, B., Yoshino, K., and Cannon. P. F. 2010. Colletotrichum: Species, ecology and interactions. IMA Fungus 1:161-165.

11. Di Rienzo, A., Peterson, A. C., Garza, J. C., Valdes, A. M., Slatkin, M., and Freimer, N. B. 1994. Mutational processes of simple-sequence repeat loci in human populations. Proc. Natl. Acad. Sci. USA 93:166-3170.

12. Evanno, G., Regnaut, S., and Goudet, J. 2005. Detecting the number of clusters of individuals using the software STRUCTURE: A simulation study. Mol. Ecol. 14:2611-2620.

13. Falush, D., Stephens, M., and Pritchard, J. K. 2003. Inference of population structure: Extensions to linked loci and correlated allele frequencies. Genetics 164:1567-1587.

14. Falush, D., Stephens, M., and Pritchard, J. K. 2007. Inference of population structure using multilocus genotype data: Dominant markers and null alleles. Mol. Ecol. Notes 7:574-578.

15. Freeman, S., Pham, M., and Rodriguez, R. J. 1993. Molecular genotyping of Colletotrichum species based on arbitrarily primed PCR, A+T-rich DNA, and nuclear DNA analyses. Exp. Mycol. 17:309-322.

16. Harrington, T. C., and Rizzo, D. M. 1999. Defining species in the fungi. Pages 43-71 in: Structure and Dynamics of Fungal Populations. J. J. Worral, ed. Kluwer Academic Press, Dordrecht, The Netherlands.

17. Huff, D. R., Peakall, R., and Smouse, P. E. 1993. RAPD variation within and among natural populations of outcrossing buffalograss Buchloe dactyloides (Nutt) Engelm. Theor. Appl. Genet. 86:927-934.

18. Hyde, K. D., Cai, L., Cannon, P. F., Crouch, J. A., Crous, P. W., Damm, U., Goodwin, P. H., Chen, H., Johnston, P. R., Jones, E. B. G., Liu, Z. Y., McKenzie, E. H. C., Moriwaki, J., Noireung, P., Pennycook, S. R., Pfenning, L. H., Prihastuti, H., Sato, T., Shivas, R. G., Tan, Y. P., Taylor, P. W. J., Weir, B. S., Yang, Y. L., and Zhang, J. Z. 2009. Colletotrichumnames in current use. Fungal Divers. 39:147-182.

19. Lu, G., Cannon, P. F., Reid, A., and Simmons, C. M. 2004. Diversity and molecular relationships of endophytic Colletotrichum isolates from the Iwokrama Forest Reserve, Guyana. Mycol. Res. 108:53-63.

20. Lubbe, C. M., Denman, S., Cannon, P. F., Groenewald, J. Z., Lamprecht, S. C., and Crous, P. W. 2004. Characterization of Colletotrichum gloeosporioides and similar species associated with anthracnose and dieback of Proteaceae. Mycologia 96:1268-1279.

21. Masel, A. M., He, C., Poplawski, A. M., Irwin, J. A. G., and Manners, J. M. 1996. Molecular evidence for chromosome transfer between biotypes of Colletotrichum gloeosporioides. Mol. Plant-Microbe Interact. 9:339348 .

22. McDermott, J. M., and McDonald, B. A. 1993. Gene flow in plant pathosystems. Annu. Rev. Phytopathol. 31:353-373.

23. McDonald, B. A. 1997. The population genetics of fungi: Tools and techniques. Phytopathology 87:448-453.

24. McDonald, B. A. 2004. Population genetics of plant pathogens. The Plant Health Instructor doi:10.1094/PHI-A-2004-0524-01

25. McDonald, B. A., and Linde, C. 2002. Pathogen population genetics, evolutionary potential and durable resistance. Annu. Rev. Phytopathol. 40:349-379.

26. McKay, S. F., Freeman, S., Minz, D., Maymon, M., Sedgley, M., Collins, G. C., and Scott, E. S. 2009. Morphological, genetic, and pathogenic characterization of Colletotrichum acutatum, the cause of anthracnose of almond in Australia. Phytopathology 99:985-995.

27. Meng, X., and Chen, W. 2001. Applications of AFLP and ISSR techniques in detecting genetic diversity in the soybean brown stem rot pathogen Phialophora gregata. Mycol. Res. 105:936-940.

28. Milgroom, M. G. 1996. Recombination of multilocus structure of fungal populations. Annu. Rev. Phytopathol. 34:457-477.

29. Milgroom, M. G., and Peever, P. T. 2003. Population biology of plant pathogens: The synthesis of plant disease epidemiology and population genetics. Plant Dis. 87:608-617.

30. Mishra, P. K., Fox, R. T. V., and Culham, A. 2003. Inter-simple sequence repeat and aggressiveness analyses revealed high genetic diversity, recombination and long-range dispersal in Fusarium culmorum. Annu. Appl. Biol. 143:291-301.

31. Moreno, M., Stenglein, S., Balatti, P., and Perelló, A. E. 2008. Pathogenic and molecular variability among isolates of Pyrenophora tritici-repentis causal agent of tan spot of wheat in Argentina. Eur. J. Plant Pathol. 122:239-252.

32. Nascimento, R. J., Mizubuti, E. S. G., Câmara, M. P. S., Ferreira, M. F., Maymon, M., Freeman, S., and Michereff, S. J. 2010. First report of papaya fruit rot caused by Colletotrichum magna in Brazil. Plant Dis. 94:1506

33. Nei, M. 1973. Analysis of genetic diversity in subdivided populations. Proc. Natl. Acad. Sci. USA 70:3321-3323.

34. Nybom, H. 2004. Comparison of different nuclear DNA markers for estimating intraspecific genetic diversity in plants. Mol. Ecol. 13:11431155.

35. OECD. 2005. Consensus document on the biology of Carica papaya L. (Papaya). No. 33. ENV/JM/MONO(2005)16.

36. Peakall, R., and Smouse, P. E. 2006. Genalex 6: genetic analysis in Excel-population genetic software for teaching and research. Mol. Ecol. Notes 6:288-295.

37. Pritchard, J. K., Stephens, M., and Donnelly, P. 2000. Inference of population structure using multilocus genotype data. Genetics 155:945-959.

38. Rampersad, S. N. 2011. Molecular and phenotypic characterization of Colletotrichum species associated with anthracnose disease of papaya in Trinidad. Plant Dis. 95:1244-1254.

39. Rodrigues, K. F., Sieber, T. N., Grünig, C. R., and Holdenrieder, O. 2004. Characterization of Guignardia mangiferae isolated from tropical plants based on morphology, ISSR-PCR amplifications and ITS1-5.8S-ITS2 sequences. Mycol. Res. 108:45-52.

40. Schlotterer, C., and Tautz, D. 1992. Slippage synthesis of simple sequence DNA. Nucleic Acid Res. 20:211-215.

41. Smith, J. M., Smith, N. H., O'Rourke, M., and Spratt, B. G. 1993. How clonal are bacteria? Proc. Natl. Acad. Sci. USA 90:4384-4388.

42. Sneath, P. H. A., and Sokal, R. R. 1973. Numerical Taxonomy. Freeman, San Francisco.

43. Sutton, B. C. 1992. The genus Glomerella and its anamorph Colletotrichum. Pages 1-26 in: Colletotrichum: Biology, Pathology and Control. J. A. Bailey and J. J. Jeger, eds. CAB International, Wallingford, UK.

44. Tamura, K., Peterson, D., Peterson, N., Stecher, G., Nei, M., and Kumar, S. 2011. MEGA5: molecular evolutionary genetics analysis using maximum likelihood, evolutionary distance, and maximum parsimony methods. Mol. Biol. Evol. 28:2731-2739.

45. Taylor, J. W., Jacobson, D. J., and Fisher, M. S. 1999. The evolution of asexual fungi: Reproduction, speciation and classification. Annu. Rev. Phytopathol. 37:197-246.

46. Teixeira da Silva, J. A., Rashid, Z., Nhut, D. T., Sivakumar, D., Gera, A., Teixeira Souza, M., Jr., and Tennant, P. F. 2007. Papaya (Carica papaya L.) biology and biotechnology. Tree For. Sci. Biotechnol. 1:47-73.

47. Ureña-Padilla, A. R., MacKenzie, S. J., Bowen, B. W., and Legard, D. E. 2002. Etiology and population genetics of Colletotrichum spp. causing crown and fruit rot of strawberry. Phytopathology 92:1245-1252.

48. Weeds, P. L., Chakraborty, S., Fernandes, C. D., d'A Charchar, M. J. Ramesh, C. R., Kexian, Y., and Kelemu. S. 2003. Genetic diversity in Colletotrichum gloeosporioides from Stylosanthes spp. at centers of origin and utilization. Phytopathology 93:176-185.

49. Weir, B. S. 1996. Genetic Data Analysis II. Sinauer, Sunderland, MA

50. Weir, B. S., and Cockerham, C. C. 1984. Estimating F-statistics for the analysis of population structure. Evolution 38:1358-1370.

51. Wolfe, A. D., and Liston, A. 1998. Contributions of PCR-based methods to plant systematics and evolutionary biology Pages 43-86 in: Plant Molecular Systematics II. D. E. Soltis, P. S. Soltis, and J. J. Doyle, eds. Kluwer Academic Press, Dordrecht, The Netherlands.

52. Yap, V. I., and Nelson, R. J. 1996. WINBOOT: A program for performing bootstrap analysis of binary data to determine the confidence limits of UPGMA-based dendrograms. IRRI Discussion Pap. Ser. No. 14. International Rice Research Institute, Manila, Philippines.

53. Yeh, F. C., and Boyle, T. B. J. 1997. POPGENE Microsoft WindowsBased Software for Population Genetic Analysis. A joint project development by Francis C. Yeh, University of Alberta and Tim Boyle, Center for International Forestry Research, Bogor, Indonesia.

54. Zhou, S., Smith, D. R., and Stanosz, G. R. 2001. Differentiation of Botryosphaeria species and related anamorphic fungi using inter simple or short sequence repeat (ISSR) fingerprinting. Mycol. Res. 105:919-926. 